\title{
Evaluation of HIV \& AIDS Surveillance System in Afghanistan
}

\author{
Hamdard Naqibullah',* and Anwar Haneef ${ }^{2}$ \\ ${ }^{1}$ Head of Afghanistan National Program for Control of AIDS, STI and Hepatitis (ANPASH), Ministry of Public Health (MoPH), Afghanistan \\ ${ }^{2}$ Lecturer, Kabul Medical University, Afghanistan
}

*Corresponding author: Hamdard Naqibullah, Head of Afghanistan National Program for Control of AIDS, STI and Hepatitis (ANPASH), Ministry of Public Health, Masood Square, Kabul, Afghanistan, Tel: 0993795590772; E-mail: Nhamdard3@hotmail.com

Received: 01 Nov, 2019 | Accepted: 18 Feb, 2020 | Published: 29 Feb, 2020

Citation: Naqibullah H, Haneef A (2020) Evaluation of HIV \& AIDS Surveillance System in Afghanistan. J HIV AIDS 6(1): dx.doi.org/10.16966/23805536.172

Copyright: (C) 2020 Naqibullah H, et al. This is an open-access article distributed under the terms of the Creative Commons Attribution License, which permits unrestricted use, distribution, and reproduction in any medium, provided the original author and source are credited.

\begin{abstract}
Afghanistan is a country with low HIV prevalence among general population. The prevalence of HIV is less than $0.1 \%$ among general population, $4.4 \%$ among People who Inject Drugs-PWID, $0.7 \%$ among prisoners, $0.4 \%$ among Men with High Risk Behavior-MHRB and $0.3 \%$ among Women with High Risk Behaviors-WHRB. To manage the response toward HIV epidemic sound data is needed but the reliable data on HIV prevalence is inadequate and not updated in the country as the last Integrated Behavioral and Biologic Survey-IBBS was conducted in 2012.
\end{abstract}

The current evaluation intends to examine the strengths of the surveillance system, pinpoints its flaws, analyze its attributes and provide recommendations for improvement.

The evaluation of HIV surveillance system in Afghanistan, conducted in line with the CDC updated guidelines for evaluating public health surveillance system from January to April 2018. Information on the operations, functionality and attributes of the HIV surveillance system was collected qualitatively using key informant interview+sessions.

Afghanistan's HIV surveillance system measures the HIV incidence in injecting drug users, men with high risk behaviors, women with high risk behaviors and prisoners. The surveillance system largely focuses on assessing the risk behaviors among these groups which lead to burst the infection.

Beside the HIV surveillance system, Afghanistan has a well-structured Health Management Information System-HMIS at national and provincial level. All health facilities report on HIV cases on monthly bases.

$91.36 \%$ of resources for surveillance system are provided by Global Fund-GF, 6.82 by World Bank-WB and $1.82 \%$ by government of Afghanistan. The heavy reliance on donors' resources poses risk to the sustainability of the system.

The HIV \& AIDS surveillance has been used to increase investment for HIV response prioritize effective interventions, program scale up, extrapolation on size of different key populations.

Data sets are accumulated in different departments and it makes it hard to obtain the overall picture of HIV response.

Spot cross-checks at service delivery sites confirmed the existence of errors in recordings and transfer of data. The reported data have details to calculate performance indicators mostly related to the proportion of clients serviced but gives limited opportunity for more in-depth analysis. The demographic variable had high level of completeness but the completeness of clinical variables (screening, diagnosis and treatment) was somewhat lower. Confidentiality was an alarming challenge. The personal identifiable information is collected in the client/patient registration forms, registration books and transferred into the reporting forms. The reporting forms are sent to the data collection unit via email which increases the possibility of data leakage. The timeliness of cases confirmation and reporting to national program varied over provinces. The mean delay between initial diagnosis and final confirmation of cases ranged from 0 to 43 days across all provinces. The delay in confirmation cases was highest in Paktika. The average participation rate by different stakeholders in weekly surveillance working group is $72 \%$ and the report submission rate is $80 \%$.

The surveillance system is pathetic to generate reliable data. The data outputs include errors. Data quality in terms of confidentiality and accuracy is limited. The system struggles to identify all new cases. In some provinces the delay in reporting is unacceptably high. The quality of data needs to be systematically improved and the data quality assessment process should be a unique part of routine supervision.

Keywords: HIV; Surveillance; Data; CDC; System attributes

\section{Introduction}

Afghanistan is a country with low HIV prevalence among general population. The prevalence of HIV is less than $0.1 \%$ among general population, 4.4\% among People Who Inject Drugs-PWID, 0.7\% among prisoners, $0.4 \%$ among Men with High Risk Behavior-MHRB and $0.3 \%$ among Women with High Risk Behaviors-WHRB. If high impact response is not maintained and scaled up the epidemic will reach concentrated level among PWIDs and 
this will help the spread of virus form key population to general population (UNAIDS, 2018) [1].

The UNODC drug report identifies that almost 1.3 to 1.6 million people who use drugs in Afghanistan in 2014 (UNODC, 2013) [2]. There here were estimated 7500 PLHIV including $28 \%$ women and 7\% children in 2017. The National AIDS Control Program- NACP reported 2800 cases (NACP, 2017) [3] of which 900 are on treatment.

The reliable data on HIV prevalence is inadequate and not updated in the country as the last Integrated Behavioral and Biologic SurveyIBBS was conducted in 2012. The current evaluation intends to examine the strengths of the surveillance system, pinpoints its flaws, analyze its attributes and provide recommendations for improvement.

\section{Methods}

The evaluation of HIV surveillance system in Afghanistan, conducted in line with the CDC updated guidelines for evaluating public health surveillance system from January to April 2018 [4]. Information on the operations, functionality and attributes of the HIV surveillance system was collected qualitatively using key informant interview sessions. The surveillance data for the time period under evaluation (2004 to 2017) was analyzed to determine the burden of the disease in the country as this is required in every surveillance evaluation.

The Key Informant Interview (KII) sessions involved 20 key stakeholders from the three-tier level of government and international partners. These key informants occupied designated positions regarding HIV/AIDS surveillance. The interview sessions were done to assess the operation, functions and attributes of the surveillance system in accordance with the CDC updated guidelines for evaluating public health surveillance system. The one-on-one interview sessions were undertaken by the lead author, who has been trained in the application of the CDC updated guideline for evaluation of public health surveillance systems by CDC Field Epidemiology and Laboratory Training Program. Informed consents were obtained from the key informants prior to the interviews. The attributes of surveillance system assessed were data quality (assessed by the presence of data supervision, data quality assurance reviews and the completeness of the data); simplicity (ascertained by the ease by which data collectors filled the form); acceptability (determined by the willingness of the surveillance data collectors and users to continue to participate in the system and rely on data from it); representativeness (assessed using the distribution of data in person and place); flexibility (assessed by retrospectively examining the ease by which the system accommodated other health events in the past decade and variation in funding and sources); sensitivity of the surveillance system (assessed based on the screening tool used for defining a case of HIV).

\section{Results}

\section{Public health importance of health outcome under surveillance}

At early stages of the epidemic, NACP collected the data from National Blood Bank-NBB and a project of International Committee of Red Cross and Red Crescent-ICRC, later, NACP included two indicators in Health Management Information System-HMIS on the number of HIV tests administered and the number of HIV cases identified. There were 892 HIV cases registered by 2007 by HMIS.

With the financial support from World Bank-WB and Global FundGF, NACP started harm reduction interventions and surveillance in 2014. The new undertaking greatly improved the case detection. For example by end of 2015, totally, 2086 HIV cases were registered. By end of 2017, 2749 HIV cases were registered and 259 new cases were added in the same year. $87 \%$ of cases had occurred between ages of 15 to 49 years. $75 \%$ cases are among men and $25 \%$ among women. The graph below describes the accumulative number of cases from 1989 to 2017 (Figure 1).

\section{HIV \& AIDS surveillance system operations}

The HIV \&AIDS surveillance system intends:

1. To quantify burden of the diseases and use surveillance data to understand in which risk group the new infections are most likely to occur.

2. To gauge the coverage of prevention and treatment services to population at risk of HIV and those who are living with HIV.

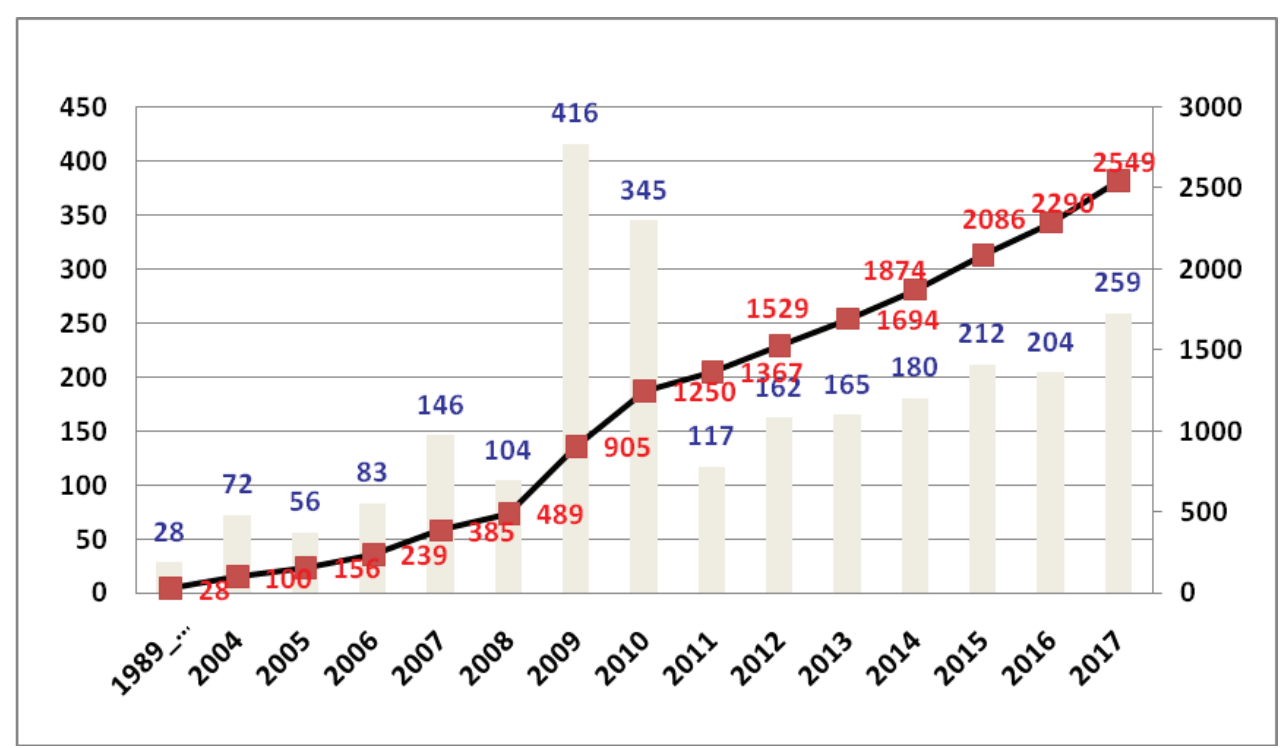

Figure 1: Accumulative number of cases from 2003 to 2017. 
The first use of surveillance data is for program planning. The planning is done both at national and sub national level to assess the magnitude and distribution of cases in different geographic areas. An understanding about distribution of epidemic helps to plan and implement harm reduction and prevention services. The second use of HIV surveillance data comprises resource allocation for example preparing procurement orders to purchase needles, syringes, condoms and medicines. The estimation for procurement orders is done using surveillance annual reports. Third use of surveillance system is to monitor the effectiveness of the interventions, for example; examining whether launching safe needle exchange programs have averted the virus transmission.

The surveillance system measures the HIV incidence in injecting drug users, men with high risk behaviors, women with high risk behaviors and prisons. The surveillance system largely focuses on assessing the risk behaviors among these groups.

Beside HIV surveillance system operated by NACP, Afghanistan has a well-structured Health Management Information System-HMIS at national and provincial level. For HIV and AIDS, the HMIS collects information routinely from Voluntary Counselling and Testing (VCT) centers and blood banks against only two indicators. The indicators are the number of HIV tests and number of people tested positive. The HMIS shares the HIV related data for each type of health facility with NACP for further utilization at end of each quarter.

There is a well-staffed surveillance unit within NACP with the clearly assigned staff roles, functions and terms of references. There are also designated surveillance managers and data officers operating partly on the intermediate level (at NGOs/sub-recipients of grants). The intermediate level is represented by the provincial coordinators who, among their other tasks, are responsible for collecting aggregated monthly reports and feedback to service-delivery level. The flow from facility level happens to national level through intermediate level with some exceptions where data goes directly from the facility to NACP (from Anti Retro Viral Treatment-ART sites, prisons and Opiate Substitute Therapy-OST centers). The diagram below summarizes the information flow (Figure 2).
But there is no designated staff to perform functions of data collection, data cleaning and verification at the service delivery sites at health facilities. Data collection is performed by the service providers (medical doctors, nurses, outreach workers, councilors, lab technicians, pharmacists, etc.) whose primary functions and capacities are laying outside of the area of data gathering. Staff who performs data registry and reporting functions usually lacks the relevant training, often do not have a clearly written instructions and overwhelmed with their primary functions where data reporting is not their priority. Most of the data are calculated manually and transferred from one form to another at the same manner, which increases the probability of errors and biases. Supervising staff at the facility level who is supposed to be reviewing the quality of data before forwarding reports to the next level have no guidelines on the process and in the best case scenario are guided by their own experience and assumptions. There is also lack of the qualified $M \& E$ managers and $M \& E$ specialists who could be able to help the data management process at intermediate or provincial level. There is limited capacity in the storage, editing and backing the data. The clients and patients are registered by their names and further on their names and other personal identifiable information are appearing at the reporting forms, which are being stored at work stations as a non-protected files, being sent back and forth and reviewed by many people who are not authorized to have an access to the personal identifiable information by international standards. There is a big risk of leakage of the personal data while being sent via electronic mail in unprotected files. There are no national guidelines or standards on maintaining the confidentiality and they need to be developed along with the introduction of the system of the unique codes for clients and patients.

\section{The resources needed to operate the HIV surveillance system}

The government of Afghanistan, Global Fund and World Bank funds the surveillance system. The telephone expenses were estimated 800 USD. The phone costs are used to support obtain information on reported cases, follow on reports and provide feedback. Other resources which are needed to operate the system include the surveillance staff incentives, stationery, travel related cost of supervision, data base
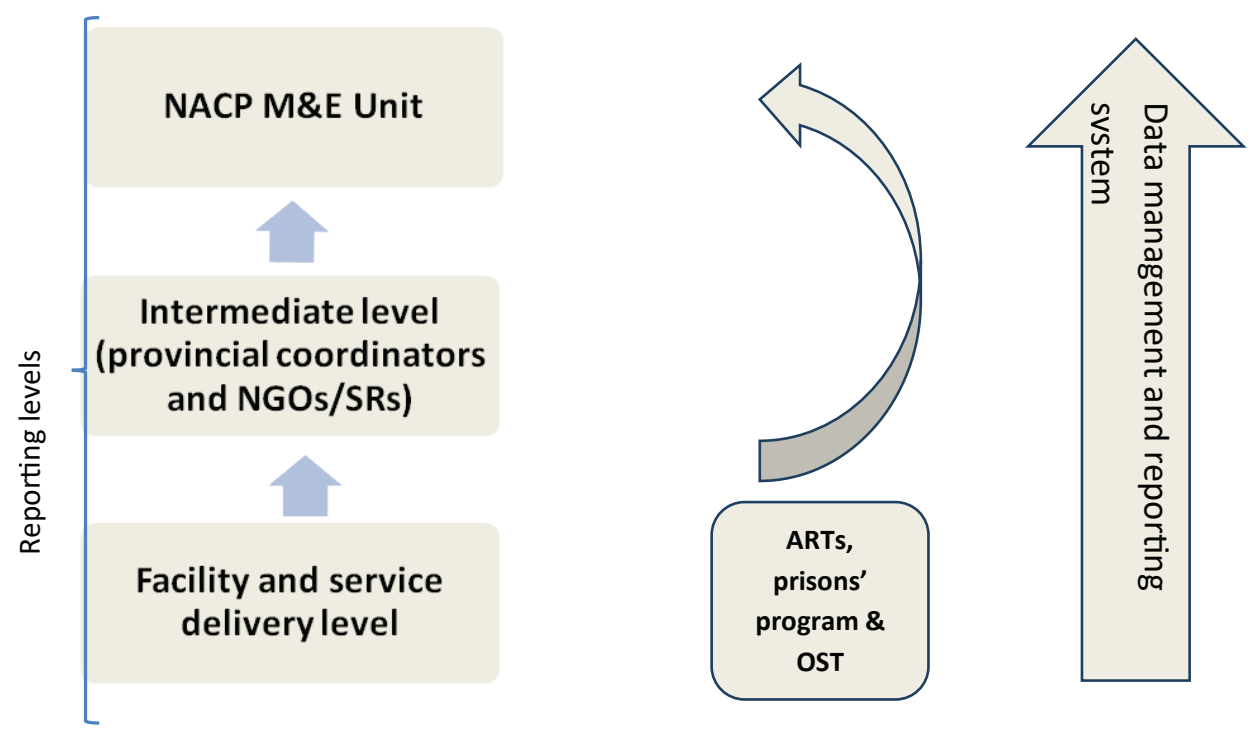

Figure 2: Reporting flow, adopted from program $\mathrm{M}$ and $\mathrm{E}$ guideline. 
infra-structure and key population size estimates. The government cover the surveillance staff salary and some travel related cost of the supervision. The overall annual operation cost of surveillance system is 344137 USD. The table below quantifies the contributions of different stakeholders (Table 1).

$91.36 \%$ of resources are provided by GF, 6.82 by World Bank and $1.82 \%$ by government of Afghanistan. The heavy reliance on donors' resources poses risk to sustainability of the system in future. The $65 \%$ of all planned resources go to Population Size Estimates-PSE. It is strategically important to know the size of population with high risk behaviors. The PSE also provides the data needed for the denominators of coverage indicators.

\section{Attributes of the HIV\& AIDS surveillance system}

Usefulness: HIV \& AIDs surveillance system is used to provide information about the incidence estimates of HIV among key populations (PWIDS, MHRBs, WHRBs and Prisoners) and to provide cross comparisons among key populations and geographic areas. Based on informal consultation with stakeholders, the HIV \& AIDS surveillance data has been used to increase investment for HIV response, prioritize effective interventions, program scale up, extrapolation on size of different key populations, provision of social services for People Living with HIV-PLHIV and ensuring treatment services for PLHIVs. The data from HIV surveillance system has the potential to be used in a variety of ways by relevant stakeholders and researchers.

Simplicity: Stakeholders who are the key consumers of the data highlighted the problem of data fragmentation and complexity of the surveillance under assessment, meaning that different data are accumulated in different departments, which makes it hard to obtain the overall picture of HIV response. From the data quality point of view it makes hard to compare and triangulate information received from different sources to identify potential problems in data reporting. The below diagram adopted from NACP reporting guideline highlights the extents of such complexity (Figure 3). For example, HIV cases related data are coming from facilities under Basic Package of Health Services and Essential Package of Health Services-EPHS, Blood Banks, Facilities under NACP and facilities operated by NGOs. Yet, the private sector facilities are not link with surveillance system.

Flexibility: The NACP data collection and report formats have been flexible to accept changes which were made based on needs at service delivery level. This has flexibility has created fragmentation and confusions. This type of flexibility increased the needs for standardized reporting formats. In 2017, NACP standardized all data collection and reporting formats in consultation with stakeholders. Now, any proposed change needs to be raised to surveillance technical working group and they will either approve or disapprove such proposals for changes based on its relevance and significance.

Data quality: The assessment visits consisted of two parts: in-depth interviews with the staff responsible for data collection and reporting and reviewing the source documents, reporting forms and tools. Data from the visited site was accessed according to seven dimensions of data quality assessment (Table 2).

Timelines: In HIV surveillance system, the new cases are reported along with monthly reports, once the "national surveillance unit" receives the monthly reports, the central database officers send out the cases detection forms to relevant sites to acquire further information about new cases. Once the cases detection forms along with service statistics are received "the national surveillance unit" undertakes data

Table 1: Operational cost of HIV surviellance system by different donors.

\begin{tabular}{|c|c|c|c|c|c|}
\hline Activity & $\begin{array}{c}\text { Estimated cost in USD } \\
\text { from WB }\end{array}$ & $\begin{array}{c}\text { Estimated cost in USD } \\
\text { from GF }\end{array}$ & $\begin{array}{l}\text { Estimated cost in USD } \\
\text { from government }\end{array}$ & Subtotal & $\%$ \\
\hline \multicolumn{6}{|l|}{ Central level } \\
\hline Telephone communication & 300 & 500 & 0 & 800 & 0.23 \\
\hline Personnel & 16520 & 33694 & 4173 & 54387 & 15.80 \\
\hline Stationary (Registers, log books) & 200 & 600 & 100 & 900 & 0.26 \\
\hline Internet & 150 & 300 & 500 & 950 & 0.28 \\
\hline Monitoring and supervision, DQA & 3000 & 6000 & 1000 & 10000 & 2.91 \\
\hline Web base database development cost & 0 & 50000 & & 50000 & 14.53 \\
\hline Key Population Size Estimation-PSE & 0 & 220000 & & 220000 & 63.93 \\
\hline \multicolumn{6}{|l|}{ Provincial level } \\
\hline Stationary (Registers, log books) & 300 & 440 & 0 & 740 & 0.22 \\
\hline Internet & 0 & 660 & 0 & 660 & 0.19 \\
\hline Sample sending & 0 & 0 & 0 & 0 & \\
\hline Travel cost related to supervision & 3000 & 2200 & 0 & 5200 & 1.51 \\
\hline Contact tracing & 0 & 0 & 500 & 500 & 0.15 \\
\hline Subtotal & 23470 & 314394 & 6273 & 344137 & 100.00 \\
\hline
\end{tabular}

Citation: Naqibullah H, Haneef A (2020) Evaluation of HIV \& AIDS Surveillance System in Afghanistan. J HIV AIDS 6(1): dx.doi. 
analysis, communicate monthly surveillance updates to a wide array of stakeholders including decision makers, and users. Additionally, the findings are discussed in surveillance technical working. The timeliness of cases confirmation and reporting to national program varied over provinces. The mean delay between initial diagnosis and final confirmation of cases ranged from 0 to 43 days across all provinces. The province specific timely cases notification ranged 0 to 6 days. The delay in confirmation cases was highest in Paktika. The table below quantifies the delay in cases confirmation and notification in 2017 (Table 3).

Acceptability: Acceptability refers to the willingness of stakeholders to participate and use the system. The HIV \& AIDS surveillance system enjoys acceptability among MoPH, NGOs, UN agencies and international partners. The average participation rate by different stakeholders in weekly surveillance working group is $72 \%$. The organizations involved in reporting are completing their reports by $80 \%$ each quarter.

Sensitivity: We defined sensitivity as the ability of the surveillance system to truly detect cases of HIV in the country (sensitivity=cases detected/existing cases $\times 100$ ). There were $7500 \mathrm{HIV}$ (True positive and True negative) cases in the country and the surveillance system detected only 2549 (True positive) by Dec 2017 so the sensitivity of HIV surveillance was calculated as $33 \%$.

Information flow received from M\&E unit of NACP (01 January 2017)

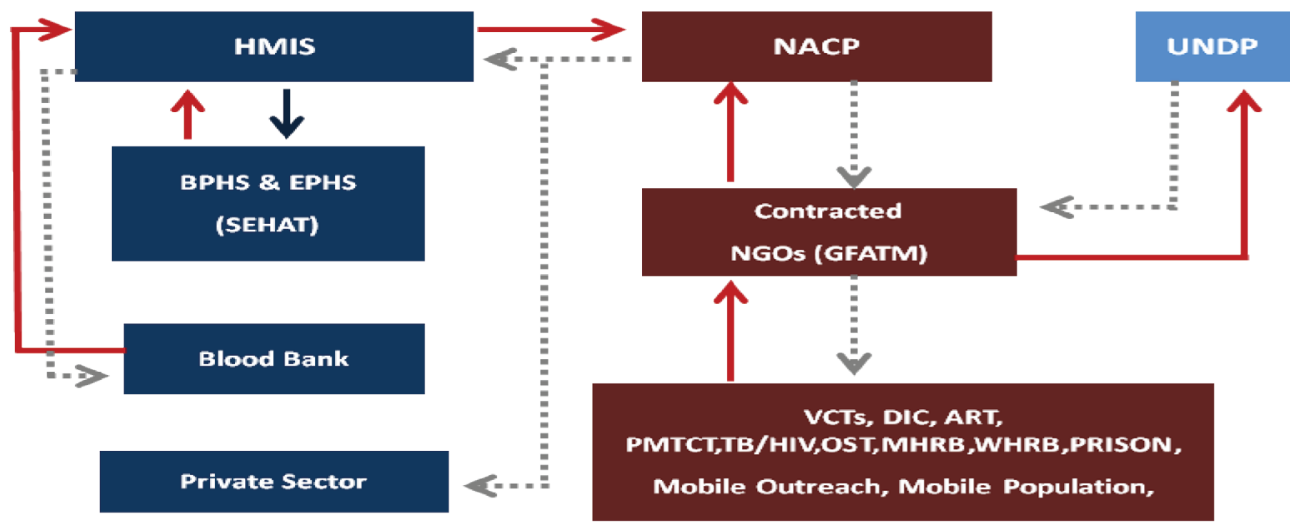

It is not clear from this diagram the reporting mechanism among Private Sector, NACP and HMIS

Figure 3: The overlap between program surveillance system and HMIS.

Table 2: Data quality dimensions of HIV surveillance system.

\begin{tabular}{|c|c|}
\hline Dimension & Results \\
\hline Accuracy & $\begin{array}{l}\text { Spot cross-checks at service delivery sites confirmed the existence of errors in recordings and transfer of data. The manual } \\
\text { transference of data from hard copies to database, absence of trained data officers at facility level and inadequate SOPs on data } \\
\text { reporting and verification contributed to occurrences of such errors. } \\
\text { The spot checks were undertaken through recalculating the "number of PLWH on ART" from monthly cohort in the source } \\
\text { document and the total reported number in the monthly report. These spot checks also included the tracing of the numbers of } \\
\text { clients served according to the quarterly report received by the intermediate level with monthly reports of the service delivery } \\
\text { site. This checks showed that data accuracy at these sites didn't not exceed } 80 \% \text {. }\end{array}$ \\
\hline Reliability & $\begin{array}{l}\text { The DIC serving women sex workers is unrealistically reporting high number of clients reached. For example, the reported numbers } \\
\text { accounted for the half of the estimated number of targets set for the whole one year only in the initial two months. The attempt } \\
\text { to cross-check the reports with the source documents (clients register books) failed due to the high level of inaccuracy in source } \\
\text { documents, which among other reasons might have led to double counting. }\end{array}$ \\
\hline Precision & $\begin{array}{l}\text { The reported data have details to calculate performance indicators mostly related to the proportion of clients serviced but gives } \\
\text { limited opportunity for more in-depth analysis. } \\
\text { For example, at treatment centers, the current reporting formats are not providing the information on any changes in the recent } \\
\text { treatment regiments, clinical success or failure, in the current format does not give a possibility to track the sero-conversion in } \\
\text { sero-discordant couples. }\end{array}$ \\
\hline Completeness & $\begin{array}{l}\text { The demographic variable had high level of completeness but the completeness clinical (screening, diagnosis and treatment) } \\
\text { variables were some lower. }\end{array}$ \\
\hline Integrity & There are no protection measures in place, data can be easily corrupted and manipulated. \\
\hline Confidentiality & $\begin{array}{l}\text { Confidentiality is an alarming challenge. The personal identifiable information is collected in the client/patient registration forms, } \\
\text { registration books and transferred into the reporting forms. The reporting forms are sent to the data collection unit via email } \\
\text { which suggests possibility of data leakage. }\end{array}$ \\
\hline
\end{tabular}

Citation: Naqibullah H, Haneef A (2020) Evaluation of HIV \& AIDS Surveillance System in Afghanistan. J HIV AIDS 6(1): dx.doi. 
Table 3: Delay in case reporting and confirmed diagnosis.

\begin{tabular}{|l|c|c|}
\hline \multicolumn{1}{|c|}{ Provinces } & $\begin{array}{c}\text { Average delay between } \\
\text { initial \& confirmed diag }\end{array}$ & $\begin{array}{c}\text { Average delay in case } \\
\text { reporting to program }\end{array}$ \\
\hline Badakhshan & 0.00 & 0.00 \\
\hline Baghlan & 0.00 & 5.00 \\
\hline Farah & 0.00 & 4.00 \\
\hline Nangarhar & 1.90 & 3.83 \\
\hline Kunar & 2.50 & 3.00 \\
\hline Kabul & 2.51 & 2.24 \\
\hline Khost & 2.83 & 3.33 \\
\hline Paktiya & 4.00 & 6.50 \\
\hline Nimroz & 7.50 & 4.25 \\
\hline Ghazni & 8.25 & 3.50 \\
\hline Herat & 9.29 & 4.07 \\
\hline Balkh & 11.31 & 2.88 \\
\hline Kunduz & 11.67 & 3.56 \\
\hline Kandahar & 33.75 & 3.65 \\
\hline Paktika & 43.00 & 0.00 \\
\hline Grand Total & 7.88 & 3.00 \\
\hline
\end{tabular}

Positive Predictive Value-PPV: PPV is the proportion of all HIV confirmed cases out of all HIV suspected cases. 1472 suspected cases were tested repeatedly for HIV 259 cases have confirmed as positive so the PPV is $5.7 \%$. This tells how good the system is to identify people with diseases and without diseases.

Representativeness: The HIV \& AIDS surveillance system only cover $40 \%$ of provinces (13 out of 34 ). The coverage is limited to key population and specific facilities in urban areas and the system doesn't cover the rural areas. The system doesn't cover general health facilities. The general health facilities are covered by HIMIS and it only reports on the number of first tests. For certain, key populations the surveillance representativeness is even more limited for example the surveillance collect information on HIV risk behavior and HIV cases among MHRBs only from five provinces (Kabul, Kandahar, Nangarhar, Mazar I Sharif and Herat). The system also doesn't include information on millions of Afghan living as refuges in Pakistan and Iran. A considerable number of population in Afghanistan seek services in private health facilities, the information from private sector is also not included in this surveillance system.

\section{Discussion}

Two parallel systems are being operated in Afghanistan. One is managed by the HMIS Unit of the MOPH and the other by the NACP under Directorate of Preventive Medicine and Communicable Disease of MOPH. HMIS collects all health related information from the government health system while the NACP collects HIV/AIDS related data predominantly from the contracted NGOs providing services for key populations in different provinces.

A well-structured HMIS has been established and functional at national and provincial level and all health facilities and BPHS implementers report on a monthly basis. For HIV and AIDS, the HMIS collects information routinely from VCT centers through BPHS system against only two indicators: number of HIV tests and number of people tested positive. The HMIS only shares the HIV related data with NACP for further utilization. All other HIV \& AIDS related information flow remains NACP responsibility HMIS is covering $85 \%$ of the country while HIV surveillance system only cover $40 \%$ of the country.
In HIV \& AIDS surveillance system, there is no clearly described process on data aggregation, analysis and steps to be performed on the each level of the reporting system. Feedback is provided to the sub-reporting levels on the quality of their reporting (accuracy, completeness, timeliness), but sporadically, most often when there are problems with the reported numbers discovered or there is a need to receive a clarification from the site. There are some elements of the quality control in place when reviewing the monthly and quarterly reporting forms, but it is not as a systematic process. At the facility level quality control is minimal, especially when the data are transferred from paper based forms into the Excel spreadsheet.

The timeliness variations across provinces resulted from different reporting protocols in the provinces (e.g., centralized versus distributed reporting within the provinces). Other factors that might have contributed to reporting delay in our study included: the patient's recognition of symptoms; the patient's acquisition of medical care; the use of confirmatory laboratory testing; reporting by the health care provider or the laboratory to the local, regional and national level; case follow-up investigations to verify the case report or to collect additional case information; periods of decreased surveillance system activity due to variable staffing levels; manual inputs of data and data processing routines, such as data validation or error checking.

Because of limited surveillance coverage, hidden key population and stigma, the case detection rate is stagnant. To increase the number of detected cases the sensitivity of surveillance system needs to be greatly improved.

Active case finding to promote early diagnosis should be considered as is a key measure to prevent onward transmission of communicable diseases and allow entry into care to individuals in need.

\section{Conclusion}

The surveillance system is weak to generate reliable data. The data outputs include errors. Data quality in terms of confidentiality and accuracy is limited. The system struggles to identify all new cases. In some provinces the delay in reporting is unacceptably high. The quality of data needs to be systematically improved and the data quality assessment process should be a unique part of routine supervision.

\section{Recommendations}

The system needs to be scaled to the whole country to give a clear picture of the epidemic and the response. There is need to implement innovative strategies including active surveillance in prisons to identify new cases. Data quality assessment needs to be incorporated as a main element of routine supervision. Problem in delay of reporting and lost to follow up should be addressed by developing sound online data base system to obtain cases reports on real time.'

\section{References}

1. UNAIDS (2018) Country factsheets. Afghanistan.

2. Islamic Republic of Afghanistan Ministry of Counter Narcotic (2013) Afghanistan Drug Report. United Nations Office on Drugs and Crime.

3. National AIDS Control Programme (2017) Annual Surveillance Update. Ministry of Public Health, Kabul.

4. Centers for Disease Control and Prevention (2017) Updated Guidelines for Evaluating Public Health Surveillance Systems. Atlanta, USA. 